\title{
Combined use of molecular techniques and capillary electrophoresis in food analysis.
}

Virginia García-Cañas, Ramón González, Alejandro Cifuentes*

Institute of Industrial Fermentations (CSIC), Juan de la Cierva 3, 28006 Madrid, Spain

* Corresponding author

acifuentes@ifi.csic.es

Tel\# 34-91-5622900

Fax\# 34-91-5644853

Keywords: DNA analysis, capillary electrophoresis, CE, CGE, PCR, PCR techniques, GMO, species identification, food chemistry. 


\begin{abstract}
In this work, the combined use of molecular techniques and capillary electrophoresis (CE) in food analysis is reviewed. An updated overview (including works published till January 2004) is presented discussing advantages and drawbacks of these combined techniques. The main applications of molecular techniques in conjunction with $\mathrm{CE}$ in food analysis include: i) species identification, ii) microbiological and toxicological analysis and, iii) detection of transgenic foods. Future outlook of this analytical methodology in food science is also provided.
\end{abstract}

\title{
GLOSSARY:
}

AFLP (Amplified Fragment Length Polymorphism), CFLP (Cleavase Fragment Length Polymorphism), CGE (Capillary Gel Electrophoresis), ERIC (Enterobacterial Repetitive Intergenic Consensus). GMO (Genetically Modified Organism), LIF (Laser Induced Fluorescence), PCR (Polymerase Chain Reaction), QC-PCR (Quantitative Competitive Polymerase Chain Reaction), RFLP (Restriction Fragment Length Polymorphism), RTPCR (Real-Time PCR), SSCP (Single-Strand Conformation Polymorphism), T-RFLP (Terminal-RFLP), UP-PCR (Universally Primed-PCR). 


\section{Introduction}

In the last years, the development and use of new analytical techniques in food science has seen a huge increase as a result of the increased concern of consumers about food and food safety. Consequently, faster, more powerful, cleaner and cheaper analytical procedures are required by food chemists, regulatory agencies and quality control laboratories to meet this demand. These analytical techniques must address an important number of issues providing information about processing; ensuring quality control and compliance with food and trade laws; or detecting adulteration, contamination or product tampering [1].

To meet these goals, food laboratories are being pushed to exchange their classical procedures for new analytical methodologies more suited to fulfill these new requirements. Thus, the interest among scientists regarding the use of the DNA present in food as analytical-target has increased dramatically for several reasons. Firstly, DNA sequences are extraordinarily suitable to provide highly specific biological information at every taxonomic level. Secondly, technologies that allow drawing this information from food samples in an easy and reliable way have evolved considerably in the last years. Namely, the development of PCR (Polymerase Chain Reaction) [2] as an analytical tool has been pivotal in this field. The sensitivity of the amplification is due to the fact that the newly synthesized sequence can be used as template in the following cycle, so that, in ideal conditions, an exponential increase in the amount of target sequence can be expected. This point is paramount when using PCR for analytical purposes since it allows exponential amplification of selected DNA sequences with a high degree of sensitivity and specificity. 
Nowadays, the availability of thermocyclers and thermostable polymerases has made possible to automate the whole process and, as a result, to popularize this technology in food research giving rise to a huge number of molecular techniques. As a clear example of this trend, the use of molecular techniques (mostly PCR-based procedures) is in part responsible of the revolution that occurred during the 1990s in food microbiology [3-5]. Thus, the use of PCR instead of conventional microbiological techniques allows obtaining fast, specific and inexpensive analysis of microorganisms at the molecular/genetic level.

On the other hand, capillary electrophoresis (CE) techniques have seen an important increase of its application in food analysis during the last years. Thus, a fast search done in Food Science and Technology Abstract (FSTA) database using as search term “capillary electrophoresis" shows that in the period 1990-1996 there were 156 papers published on this topic; in the same number of years during 1997-2003 the search gave 482 publications, demonstrating that CE is being more and more used for food analysis.

The combined use of molecular techniques together with CE can, therefore, bring about an impressive analytical procedure that combines the selectivity and sensitivity increase provided by any molecular technique with the speed of analysis, resolving power and low sample requirements of CE techniques. These advantages can be very useful in many application fields, including clinical, forensic, pharmaceutical, and, the subject of this review, food analysis. Thus, the PCR-based techniques combined with capillary electrophoresis separation have demonstrated to be a powerful analytical alternative for: i) species identification, when fraudulent substitution, addition or contamination are suspected in foodstuff; ii) detection of food-borne pathogens; and iii) detection of 
genetically modified organisms (GMOs). Nowadays, these applications have important health, religious and/or economic implications for consumers, food industry and/or regulatory agencies and they are described below.

\section{Species identification.}

Species identification of animals or plants is a difficult task when they have lost the morphological characters (shape, size, appearance, etc.) due to food processing. For that reason, species identification methods are generally based on detection of speciesspecific compounds such as proteins and DNA [6,7]. Although some protein-based methods have been successfully developed for species identification in raw material, species distinction becomes difficult in complex matrices or heat-treated samples [8]. In contrast, species identification by DNA-based methods offers interesting advantages due to the stability of DNA at high temperatures and its more uniform distribution in the different tissues of the organism.

\subsection{Dairy products.}

Several reasons (as e.g., food safety, economical impact or consumers health) are behind the need of species identification. For instance, the increasing number of people suffering from allergy makes necessary the development of analytical methods able to identify in a sensitive and reliable way species that can be responsible of the apparition of allergens in foods. This is the case, for instance, of ß-lactoglobulin a whey protein that can act as a potent allergen and that can be unexpectedly found in infant formulas and other dairy products, due to unfair manufacture practices. 
Recently, T-RFLP has successfully been used to detect different animal species in dairy products [9]. Using a derivatized primer with HEX fluorophore results in amplification products labeled at one end that, after digestion with a restriction endonuclease, render differently sized DNA fragments, depending of the actual restriction map (i.e., which in turns depends on the species). These end-labeled restriction fragments are analyzed using high resolution capillary gel electrophoretic for accurate sizing. The procedure has been successfully used for the distinction of goat, sheep, buffalo and cow milk in dairy products [9].

\subsection{Meats.}

Nowadays, there is a growing need of new analytical procedures able to detect adulteration practices, including the fraudulent substitution of the more expensive meats for cheaper meats, or even non-meat protein additions. In addition to economic, religious and legal aspects, these practices are the object of public concern due to the risk of allergic responses or, in some cases, the more dangerous risk of transmission of certain zoonotic diseases to man, like bovine spongiform encephalopathy or avian flu. These concerns have promoted the development of new analytical tools for identification of animal and plant species in meat and in processed meat-derived products for human and animal consumption [10].

A combination of PCR-RFLP (PCR-based restriction fragment length polymorphism) and capillary gel electrophoresis with fluorescence detection has been exploited in order to differentiate species of commercial interest from others that could be fraudulently introduced. Thus, the use of CGE instead of traditional slab gel electrophoresis has 
allowed the detection of shorter DNA fragments (down to $40 \mathrm{bp}$ ), and sensitivity lower than $1 \%$ beef contamination in heat-treated pork meat (see Figure 1) [11].

\section{Microbiological food analysis.}

Microbiological food analysis has been traditionally based on the growth, isolation and morphological and physiological characterization of microorganisms. However, these methods present several limitations for their adequate application to modern production processes, and can not give rapid responses to outbreaks of food-associated infections. The application of DNA-based detection and quantification methods overcomes some of these limitations, sometimes by making unnecessary the growth and isolation of microorganisms, and sometimes by speeding up the process of species and/or strain identification.

\subsection{Food-borne pathogens.}

Genotyping of Salmonella spp. isolates is a powerful tool for the differentiation of strains and could help to trace the origin of contamination and to give appropriate responses in case of outbreaks. AFLP with fluorescent labeled primers and CGE showed a discriminatory power comparable to that of pulsed-field gel electrophoresis, obtaining fingerprint DNA patterns with \pm 1 bp resolution in runs of about 30 minutes versus 22 hours (and radioactive labeling) for conventional sequencing gel electrophoresis [12]. Although AFLP was originally developed with conventional sequencing gels and radioactive labeling, the use of CGE and fluorescence detection has been shown to greatly simplify the procedure [13]. 
Listeria monocytogenes is one of the so-called emergent food-borne pathogens with a high mortality rate among susceptible people like children, pregnant women or immuno-compromised individuals. Listeria detection and identification by traditional microbiological methods is time and labor consuming due to its low growth rates and special culture requirements. PCR methods have been successfully applied to Listeria detection/identification and to Listeria strain identification. Thus, L. monocytogenes strain identification has been reported based on ERIC (Enterobacterial Repetitive Intergenic Consensus) fingerprints resolved by CGE increasing in this way sensitivity, resolution, and DNA sizing accuracy [14].

T-RFLP using two labeled PCR primers (with HEX and 6-FAM dyes) has been applied to analyze pathogens in fish. The method consists in amplification of a highly conserved region of the $16 \mathrm{~S}$ gene with a primer pair labeled at the 5'-end with the fluorescent dyes (HEX and 6-FAM), a subsequent digestion with restriction enzymes and separation of DNA fragments using CGE-LIF. This T-RFLP method allowed detection of Vibrio, Aeromonas and Flovobacterium species. The assay was demonstrated to be useful to detect cells of Flavobacterium psychrophilum on fish kidney tissue, with a sensitivity of 30 cfu of Flavobacterium psychrophilum per mg of tissue [15].

\subsection{Toxin producing microorganisms.}

The presence in foods of some specific microorganisms is undesirable because of the toxins they can produce rather than for their direct effects on human health. Nevertheless their specific detection is still interesting, because it is indicative of the risk of the presence of the toxin, and can greatly contribute to food safety. This is the case for Clostridium botulinum, an anaerobic spore-forming bacterium that is 
widespread in the environment. Neurotoxins are the main factors responsible for all symptoms and lesions observed in the disease called botulism. Identification of $C$. botulinum in food can be performed by classical methods of bacteriology including culture in anaerobic conditions. However, more sophisticated methods involve the identification of $C$. botulinum strain by means of toxin genes detection. The identification of $C$. botulinum strains carrying genes coding for botulinic toxin A, B, E, F, or G using specific primers and specific DNA probes or nested PCR systems for each toxin gene has been described by several authors [16]. Moreover, an analytical alternative using CGE to detect the neurotoxin gene has been described. Using lowviscosity entangled polymer buffer as molecular sieving, the separation took place in 17 minutes, demonstrating a greater range in DNA size separation and better sensitivity than slab gel electrophoresis [17]. Rapid identification of the specific botulinic toxin responsible of food-poisoning may help to take quick and correct decisions for antidote and treatment.

Enterohemorragic Escherichia coli (O157:H7) produces verotoxin that has two subunits (A and B) and interfere with protein synthesis process in the cell. There are two versions of the verotoxin (VT1 and VT2) with similar activity and its detection is important to prevent gastrointestinal syndrome. Advantages of combining CGE with three molecular techniques (allele specific PCR, SSCP and CFLP) to develop rapid and specific analysis of the VT1 and VT2 toxin genes of E. coli (O157:H7) have been studied [18]. Allele specific duplex PCR-CGE analysis showed separated peaks corresponding to $174 \mathrm{bp}$ fragment VT1 and 128 bp fragment VT2 in 4 minutes (see Figure 2), with coefficients of variation for migration times of 0.1 and $0.29 \%$ for VT1 $(n=6)$ and VT2 (n=5), respectively, and a detection limit of 50 fg of genomic DNA 
(approximately 100 bacteria). SSCP-CGE method using capillaries of $30 \mathrm{~cm}$ of effective length, $20^{\circ} \mathrm{C}$ and $7.4 \mathrm{kV}$ showed good separations of VT1 and VT2 in 17 minutes. Finally, CFLP-CGE method showed coefficients of variation within-assay and between-assay equal to 0.27 and $0.35 \%(n=5)$, respectively, for bacteria containing two VT1/VT2 genes in runs of 6 minutes. The excellent results in terms of reproducibility and speed of analysis make the three methods suitable for verotoxin gene detection purposes.

\section{Detection of transgenic foods.}

Genetic engineering is used in agriculture and food industry in order to improve: i) the performance of plant varieties (resistance to plagues, herbicides, and hydric or saline stresses), ii) technological properties during storage and processing (firmness of fruits), and/or iii) the sensorial and nutritional properties of food products (starch quality, content on vitamins or essential amino acids) $[19,20]$.

Commercial use of transgenic plants and other genetically modified organisms (GMOs) has raised several ideological and ethical issues during the past few years. The debate is especially intense, for several reasons, in the case of the so-called "transgenic foods". For instance, placing in the market food or feed consisting or containing GMOs is subject to compulsory labeling in the European Union (EU) [21]. Because an adventitious contamination of GM-material in a non-GM background is difficult to avoid, and labeling as "GMO-containing” could severely affect the marketing of food products, EU regulations have fixed a $0.9 \%$ threshold for adventitious contamination that is not subject to labeling requirement. This has created a demand for analytical methods that can detect and quantify the amount of GMO in foods [22]. 
GMOs could be detected both by PCR, for direct detection of the transgenic DNA, or by immunological methods, for detection of the cognate proteins (limited to tissues in which the transgene is expressed) [22]. PCR methods are usually preferred for GMO detection due to their better reliability and sensitivity and to the higher stability of DNA over proteins. Although RT-PCR is nowadays gaining popularity over QC-PCR for the quantitation of GMOs in food samples [23,24], these methods are still under development for the simultaneous detection of several transgenes. Besides, the interlaboratory reproducibility of RT-PCR is too low as it has been demonstrated by the high \%RSD values frequently obtained (e.g., 40\% [25] or 33.4\% [26]).

The combined use of PCR techniques and CGE seems to be a good alternative for the detection and quantitation of transgenic organisms in foods. Thus, in the first work published on the use of CGE to detect transgenic foods [27], a new CGE method was developed that allowed obtaining reproducible separations of DNA fragments using commercially available polymers together with bare fused silica capillaries. The method combined a washing routine of the column with $0.1 \mathrm{M}$ hydrochloride acid followed by a rinsing step with a dissolution containing 1\% polyvinyl alcohol. The use of this procedure together with a running buffer containing 2-hydroxyethyl cellulose (HEC) gave highly resolved separations of DNA fragments ranging from 80 to $500 \mathrm{bp}$. The separation of these DNA fragments was achieved in ca. 20 minutes with efficiencies up to $1.8 \cdot 10^{6}$ plates/m and high intra- and inter-day reproducibility. The length up to 500 bp corresponds to the DNA sizes more frequently amplified by PCR for detecting GMOs in foods. 
In a following work [28], some useful considerations regarding optimization of DNA extraction from maize flour were given. Four different methods for extraction of DNA were compared from which SDS/proteinase $\mathrm{K}$ method was chosen as the most convenient. DNA samples extracted from maize flour were then amplified by PCR. To do this, a test fragment of the "foreign" $\operatorname{cryIA}(b)$ gene (GenBank accession number I41419) was amplified using primers cryIA(b)-V3 and cryIA(b)-V4 [28]. Amplification of a fragment of the maize starch synthase gene dull1 (a natural gene in maize), used as a control for DNA quality and amplificability, was performed with primers MSS-S and MSS-A [28]. The DNA amplified by PCR was next injected into the CGE-UV equipment. Although the sensitivity of the PCR-CGE-UV procedure was enough to detect $1 \%$ of transgenic maize in food samples, the peak obtained for the amplified DNA was too close to the detection limit and, therefore, an optimization of the sensitivity of this method was carried out by using CGE with laser induced fluorescence (LIF).

The use of LIF improves dramatically both the limit of detection and the linear dynamic range obtainable in CGE compared with UV detection. Basically, there are two procedures to supply fluorescence to DNA fragments when excited with an $\mathrm{Ar}+$ laser (usually $\lambda_{\mathrm{ex}}=488 \mathrm{~nm}$ ). The first one is based on covalently binding the DNA molecules with a derivatizing agent (containing frequently fluorescein). The second one uses intercalating dyes (for double-stranded dsDNA) added to the buffer as e.g., ethidium bromide (EtBr), thiazole orange (TO), oxazole yellow (YO), or their corresponding homodimers, that form stable fluorescent complexes when bound to dsDNA fragments. Ultrasensitive detection of genetically modified maize DNA could be achieved by CGELIF using different fluorescent intercalating dyes [29]. To do that, four different 
fluorescent intercalating dyes were compared for the CGE-LIF detection of DNA from transgenic maize in flours (see Table 1). It was demonstrated [29], that the use of YOPRO-1 as fluorescent intercalating dye provided optimum conditions in terms of sensitivity (i.e., LOD was 1000 zmol, calculated for a 200 bp DNA fragment), efficiency (up to $2.4 \times 10^{6}$ plates/m), speed of analysis, reproducibility and cost per run. Using this fluorescent compound, the fluorescence signal was shown to vary linearly with the DNA concentration in the range studied, i.e., $1-500 \mathrm{ng} / \mu \mathrm{l}$, what was a good indication of the quantitative possibilities of this analytical procedure. It was demonstrated that using this method, $0.01 \%$ of transgenic maize could be detected in flour by direct injection of the PCR amplified sample; an example of this PCR-CGELIF analysis is given in Figure 3.

In a recent work [30], a new procedure useful for quantitative analysis of GMOs in foods has been described and applied to analyze transgenic Bt Event-176 maize. The method developed is based on the co-amplification of specific DNA maize sequences with internal standards using quantitative competitive PCR (QC-PCR). The QC-PCR products were quantitatively analyzed using the CGE-LIF method described above [29]. The CGE-LIF procedure allowed using internal standards differing by only 10 bp from the original target fragments, to our knowledge, the smallest size difference that can be found in the bibliography for QC-PCR of GMOs. It was shown that CGE-LIF provided better resolution and a signal/noise ratio improvement of ca. 700 -fold compared to slab gel electrophoresis (see Figure 4). The good possibilities in terms of quantitative analysis of GMOs provided by this new method were confirmed by determining the $\mathrm{Bt}$ Event-176 maize content in different certified reference maize powders and food samples of known composition. 
Recently, the good possibilities of a commercial microfluidic capillary electrophoresis system (LabChip ${ }^{\mathrm{TM}}$ ) have been demonstrated for the analysis of GMOs in foods [31, 32]. It is demonstrated that the use of microfluidic systems offer improvements in quantitation accuracy, objectivity and ease of use compared with traditional agarose slab gel electrophoresis.

\section{Conclusions and future outlook.}

The combined use of molecular techniques and CGE is currently increasing as a result of its good possibilities in terms of selectivity, sensitivity and speed of analysis. Thus, it is expected that in the non-distant future its use in food science (and more specifically for species identification, microbiological analysis or detection of transgenic foods) become routinely applied. Moreover, some other food applications that can take advantage of the capabilities of this combination of methodologies can be anticipated, as for instance, monitoring of standard and modern food processes or following the development of the so called "new foods".

There are very few works reporting the combined use of PCR amplification and CGE to detect food-borne pathogens. The higher sensitivity and potential to distinguish very similar strains of the same species makes PCR-CGE very suitable for fast studies of microbial populations in molecular epidemiology.

The apparition in the market of new foods and food products containing GMOs will maintain the need for new analytical methods allowing for a qualitative and, mainly, quantitative analysis of these GMOs. Currently, there are several combinations of 
amplification, detection and quantification technologies available for GMO detection [22], but very few of them have passed through a validation process in order to be confidently used by enforcement and commercial laboratories. In order to provide the control organisms with the appropriate tools, and given the path of the evolution of the GMO market, future research in this field will keep focusing on methods able to reliably detect and quantify several different GMOs in a single analysis, where the combined use of competitive quantitative-PCR and CGE can play an important role.

\section{Acknowledgements}

VGC thanks to Consejería de Educación y Cultura (Comunidad de Madrid) for a fellowship. Authors also thank to Comunidad de Madrid for supporting this work with the project 7B-0021-2002. 


\section{References}

[1] E. Ibañez, A. Cifuentes, Crit. Rev. Food Sci., 41 (2001) 413.

[2] K. Mullis, F.A. Fallona, Method. Enzymol., 155 (1987) 335.

[3] W.E. Hill, Crit. Rev. Food Sci. Nutr., 36 (1996) 123.

[4] D.W. Pimbley, P.D. Patel, J. Appl. Microbiol., 84 (1998) 98S.

[5] P.M. Scheu, K. Berghof, U. Stahl, Food Microbiol., 15 (1998) 13.

[6] R. Meyer, U. Candrian, Lebensm. Wiss. Technol., 29 (1996) 1.

[7] C.G. Sotelo, R.I. Pérez-Martín, in: Food authenticity and traceability, M. Lees (Ed.), CRC Press, Boca Raton, Washington, US., 2003, pp. 323-346.

[8] C. Wolf, M. Burgener, P. Hübner, J. Lüthy, Lebensm. Wiss. Technol., 33 (2000) 144.

[9] I. Lanzilao, F. Burgalassi, E. Ebranati, L. Cioni, R. Fani, S. Fancelli, M. Settimelli, Industrie Alimentari, XLII (2003) 595.

[10] P. KrcMar, E. Rencova, J. Agr. Food Chem., 51 (2003) 7655.

[11] Y. Sun, C. Lin, J. Agr. Food Chem., 51 (2003) 1771.

[12] B. Lindstedt, E. Heir, T. Vardund, G. Kapperud, J. Clin. Microbiol., 38 (2000) 1623.

[13] C. Knorr, H.H. Cheng, J.B. Dodgson, Anim. Genet., 30 (1999) 28.

[14] C. Sciacchitano, Electrophoresis, 19 (1998) 66.

[15] W. B. Nilsson, M. S. Strom, Dis. Aquat. Organ., 48 (2002) 175.

[16] M.R. Popoff, in: PCR Detection of microbial pathogens, K. Sachse, J. Frey (Ed.), Humana Press, New Jersey, US, pp. 137-152.

[17] C. Sciacchitano, I.N. Hirshfield, J. AOAC Int., 79 (1996) 861.

[18] H. Arakawa, K. Watanabe, H. Kashiwazake, M. Maeda, Biomed. Chromatogr., 16 (2002) 41. 
[19] M.G. Koziel, G.L. Beland, C. Bowman, N.B. Carozzi, R. Crenshaw, L. Crossland, J. Dawson, N. Desai, M. Hill, S. Kadwll, K. Launis, K. Lewis, D. Maddox, K. McPherson, M.R. Meghji, E. Merlin, R. Rhodes, G.W. Warren, M. Wright, S.V. Evola, Biotechnology, 11 (1993) 194.

[20] A.G. Gao, S.M. Hakimi, C.A. Mittanck, Y. Wu, H. Woerner, D.H. Stark, D.H. Shah, J. Liang, C.M. Tommens, Nature Biotech., 18 (2000) 1307.

[21] Regulation (EC) No 1830/2003 of the European Parliament and of the Council (22 September 2003).

[22] V. García-Cañas, A. Cifuentes, R. González, Crit. Rev. Food Sci., in press.

[23] M. Vaïtilingom, H. Pijnenburg, F. Gendre, P. Brignon, J. Agr. Food Chem., 47 (1999) 5261.

[24] K.G. Berdal, A. Holst-Jensen, Eur. Food Res. Technol. 213 (2001) 432.

[25] M. Höhne, C.R. Santisi, R. Meyer, Eur. Food Res. Technol., 215 (2002) 59.

[26] A. Pardigol, S. Guillet, B. Pöpping, Eur. Food Res. Technol. 216 (2003) 412.

[27] V. García-Cañas, R. González, A. Cifuentes, J. Sep. Sci., 25 (2002a) 1.

[28] V. García-Cañas, R. González, A. Cifuentes, J. Agr. Food Chem., 50 (2002b) 1016.

[29] V. García-Cañas, R. González, A. Cifuentes, J. Agr. Food Chem., 50 (2002c) 4497.

[30] V. García-Cañas, A. Cifuentes, R. González, Anal. Chem. 76 (2004) 2306.

[31] L. Birch, C.L. Archard, H.C. Parkes, D.G. McDowell, Food Control, 12 (2001) 535.

[32] M. Burns, D. Shanahan, H. Valdivia, N. Harris, Eur. Food Res. Technol., 216 (2003) 428. 
Table 1. Characteristics of four different fluorescent intercalating dyes used to detect dsDNA by CGE-LIF using an Ar+ laser [29].

\begin{tabular}{lccccc}
\hline & LOD $^{\mathbf{a}}$ & Analysis time $^{\mathbf{b}}$ & Buffer renewal $^{\mathbf{c}}$ & Efficiency $^{\mathbf{d}}$ & Cost $^{\mathbf{e}}$ \\
\cline { 2 - 6 } SYBR-Green-I & 700 & 47 & After 3 runs & 1600000 & 0.25 \\
YOPRO-1 & 1000 & 30 & After 5 runs & 2400000 & 0.04 \\
EnhanCE & 11300 & 29 & After 4 runs & 2400000 & 0.29 \\
EthBr & 97400 & 34 & After 5 runs & 2700000 & 0.003 \\
\hline
\end{tabular}

${ }^{a}$ limit of detection in zmol for the 200 bp fragment

$\mathrm{b}$ analysis time in min for the separation of DNA fragments from 80 to 1000 bp

${ }^{\mathrm{c}}$ calculated for a $2 \mathrm{ml}$ vial of running buffer

d number of theroretical plates per meter of column for the $200 \mathrm{bp}$ fragment

${ }^{\mathrm{e}}$ cost per run in US\$ 


\section{FUGURE LEGENDS}

Figure 1. Electrophoregrams of PCR-RFLP products amplified from mitochondrial 12S rRNA gene of pork, goat, and beef. The fluorescent labeled PCR products are digested by DdeI and then separated using CGE. Redrawn from ref [11].

Figure 2. Separations of O157 verotoxin genes (VT1, VT2 and VT1/VT2) obtained by allele-specific PCR. Electrophoregrams of allele-specific PCR products (174 bp for VT1 and 128 bp for VT2) were obtained from VT1, VT2 and VT1/VT2 genecontaining O157 DNAs. Separation conditions were 3\% polyacrylamide, running temperature at $25^{\circ} \mathrm{C}$, voltage at $6.4 \mathrm{kV}$, capillary effective length: $7 \mathrm{~cm}$, fluorescence dye: thiazole orange $0.1 \mu \mathrm{g} / \mathrm{mL}$. Redrawn from ref [18].

Figure 3. CGE-LIF electrophoregrams obtained for the PCR amplification reactions from: A) $0.01 \%$ transgenic maize DNA using the primer pair MSS-S/MSS-A; B) $0.01 \%$ transgenic maize DNA using the primer pair cryIA(b)-V5/cryIA(b)-V6; C) conventional maize DNA using the primer pair cryIA(b)-V5/cryIA(b)-V6. Samples injected for $12 \mathrm{~s}$ using $\mathrm{N}_{2}$ pressure (1 psi). Redrawn from ref [29].

Figure 4. Electrophoretic analysis of a series of QC-PCR reactions (cryIA(b) system) with $0.21 \mathrm{fg}$ of pBTIS internal standard per reaction. The analysis was performed by A) slab agarose gel or B) CGE-LIF. Samples: M) DNA molecular weight marker; 1) control in the absence of DNA template, 2) control in the absence of internal standard, 3- 8) competitive reactions with increasing amounts of transgenic Bt Event-176 maize genomic DNA: 3) 2.5 ng; 4) 0.77 ng; 5) 0.26 ng; 6) 0.12 ng; 7) 0.04 ng; 8) 0 ng) per reaction. Redrawn from ref [30]. 


\section{Biographical details:}

Virginia García-Cañas was born and raised in Madrid. After receiving her Master Degree in Biology (1996, Alcala de Henares University), she obtained her Master Degree in Food Science and Technology (1998, Autonoma University of Madrid). In 1999, Autonoma University of Madrid rewarded her with the "Extraordinary Master Degree" award. In 2000, she embarked on the research world at the Industrial Fermentations Institute (CSIC) as PhD student under the supervision of Dr. Cifuentes and Dr. González. Nowadays, she participates in the research project "Multiple, ultrasensitive and quantitative detection of GMOs in foods" supported by CAM. She is specially interested in the detection and quantitation of GMOs and food pathogens by means of the combined application of capillary electrophoresis and polymerase chain reaction techniques (PCR).

$* * * * * * * * * * * * * * * * * * * * * * * * * * * * * * * * * * * * * * * * * * * * * * * * * * * * *$

Dr. Ramón González is a tenured scientist at the Institute of Industrial Fermentations, CSIC, Madrid, Spain. He received his Masters Degree in Biology in 1989 (University of Valencia), and his Doctorate in Molecular Biology in 1993 (University of Valencia), working in the Institute of Agro-chemistry and Food Technology in the field of Microbial Biotechnology. From 1994 to 1998 he specialized in Microbial Genetics working as an EMBO post-doctoral fellow and as a Marie Curie post-doctoral fellow at the Institute for Genetics and Microbiology (University of Paris XI/CNRS). Currently he performs active research in the field of Microbial and Enzymatic Biotechnology, and developing DNA-based Methods for Food Analysis.

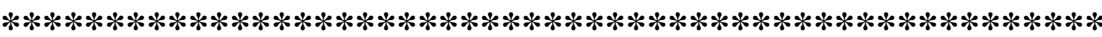

Dr. Alejandro Cifuentes is a senior researcher at Institute of Industrial Fermentations, (CSIC) in Madrid, Spain. He received his Masters Degree in Chemistry in 1988 (University of Valladolid), and his Doctorate in Chemistry in 1993 (University of Valladolid). He was working as a Marie Curie post-doc fellow at the University of Amsterdam from 1993 to 1995. In 1999, he successfully sat the exam for a staff position (Científico Titular) at CSIC. In 2001 and 2002 he was appointed Honoray Professor of the University Autonoma of Madrid and in 2003 he was promoted to Senior Researcher (Investigador Científico). Currently, he leads the following research lines: i) Multiple detection and quantitation of genetically modified organisms (GMOs, transgenic foods) and food pathogens by PCR-CGE-LIF; ii) chiral molecules as food-quality markers analyzed by CE; iii) capillary electrophoresis-mass spectrometry applied for proteomics, food quality and food safety; iv) chemical and biological characterization of functional ingredients from natural sources (plants, alga and microalga); v) monitoring new drug delivery systems using $\mathrm{CE}$ techniques. 